\title{
Evaluation of Rheometry Amplitude Sweep Cross-Over Point as an Index of Flexibility for HA Fillers
}

\author{
Åke öhrlund \\ Galderma, Uppsala, Sweden \\ Email: ake.ohrlund@galderma.com
}

How to cite this paper: Öhrlund, $\AA$. (2018) Evaluation of Rheometry Amplitude Sweep Cross-Over Point as an Index of Flexibility for HA Fillers. Journal of Cosmetics, Dermatological Sciences and Applications, 8, 47-54.

https://doi.org/10.4236/jcdsa.2018.82008

Received: March 23, 2018

Accepted: June 18, 2018

Published: June 21, 2018

Copyright $\odot 2018$ by author and Scientific Research Publishing Inc. This work is licensed under the Creative Commons Attribution International License (CC BY 4.0).

http://creativecommons.org/licenses/by/4.0/

\begin{abstract}
Background: The majority of studies published on rheology of hyaluronic acid (HA) fillers have focused on results from frequency sweeps within the linear viscoelastic region (LVR), i.e. at nearly static conditions. To study the properties and behavior of HA fillers at more dynamic conditions, it is necessary to go outside the LVR. Objective: Evaluation of the G'/G" cross-over point from the amplitude sweep is suggested as a manner to estimate the flexibility of HA fillers. Materials and methods: Rheological properties of $7 \mathrm{HA}$ fillers were measured in an amplitude sweep from $0.1 \%$ to $10000 \%$ strain at $1 \mathrm{~Hz}$, using an Anton Paar MCR 301, a PP-25 measuring system with a gap of $1 \mathrm{~mm}$ at $25^{\circ} \mathrm{C}$ and a $30-\mathrm{min}$ relaxation time. The cross-over point was evaluated for stress, strain and G (G' and G' identical), the values denoted xStrain, xStress and $\mathrm{xG}$. Results: The $\mathrm{xStrain}$ values spanned from below $1000 \%$ to above $2000 \%$ for the products based on the Optimal Balance Technology (OBT) ${ }^{\mathrm{mm}}$ (in the US, XpresHAn Technology $\left.\mathrm{y}^{\mathrm{Tn}}\right)$, compared to below $100 \%$ for the products based on the NASHA ${ }^{\oplus}$ technology. Conclusions: Measurement of the flexibility provides a more complete picture of the rheological properties of HA fillers as a complement to firmness measured as G'. The test results show that the Restylane family of products covers a large range in flexibility, and that the flexibility can be estimated using xStrain derived from the amplitude sweep.
\end{abstract}

\section{Keywords}

HA Fillers, Rheology, xStrain, Flexibility

\section{Introduction}

The first hyaluronic acid (HA) filler registered in the US was Restylane in 2003. Since then, the market has seen an ever increasing growth, with more than a 
hundred products available in Europe today. This abundance makes selecting the appropriate product suitable for each patient, indication and injection technique a challenging task. To aid in that task, scientific differentiation of products based on their physicochemical properties have drawn attention. One of the popular and most accepted methodologies in this aspect has been rheology. The resistance to deformation, the firmness, measured as the elastic modulus $\mathrm{G}^{\prime}$, is often suggested to act as a basis for trying to understand how physical properties of an HA filler relates to its in vivo performance [1]-[13].

The determination of $\mathrm{G}^{\prime}$ is performed under nearly static conditions, i.e. applying very small deformations during the rheological measurement. This is in order to fulfill the requirement to keep within the linear viscoelastic region (LVR), the region where the stress changes linearly with deformation. These measurements are normally performed as a frequency sweep, where the amount of deformation is kept constant while increasing the frequency (Figure 1(a)).

It is certainly in order to consider the physical properties of an HA filler under these conditions, since a large part of the fillers life-time in the tissue will pass in static or near static conditions. Some of the time in the tissue, however, the HA filler will experience movement, flexing and stretching, due to e.g. facial expressions. Therefore, it is interesting also to study the properties under more dynamic conditions.

Such conditions apply during an amplitude sweep, where the amount of deformation (strain) is increased (Figure 1(b)) until a change in the signal is observed. An amplitude sweep is performed in order to verify that the amount of deformation used for the frequency sweep is within LVR. Results from a frequency sweep and an amplitude sweep of an HA filler are depicted in Figure 2.

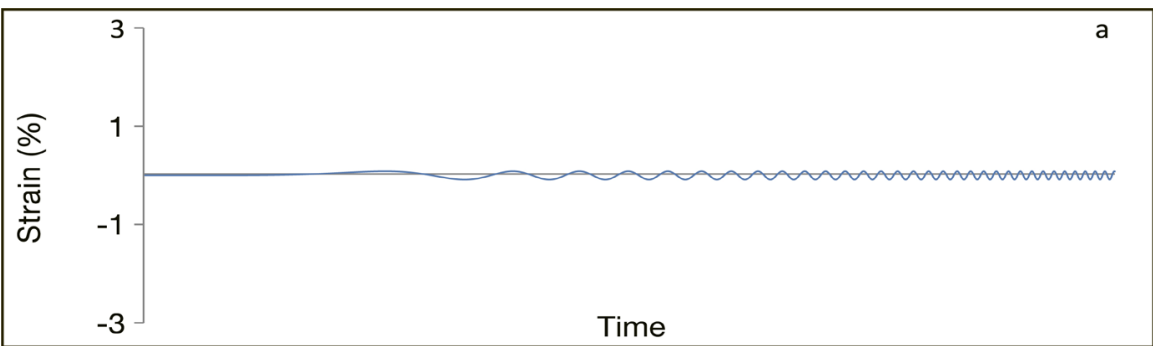

(a)

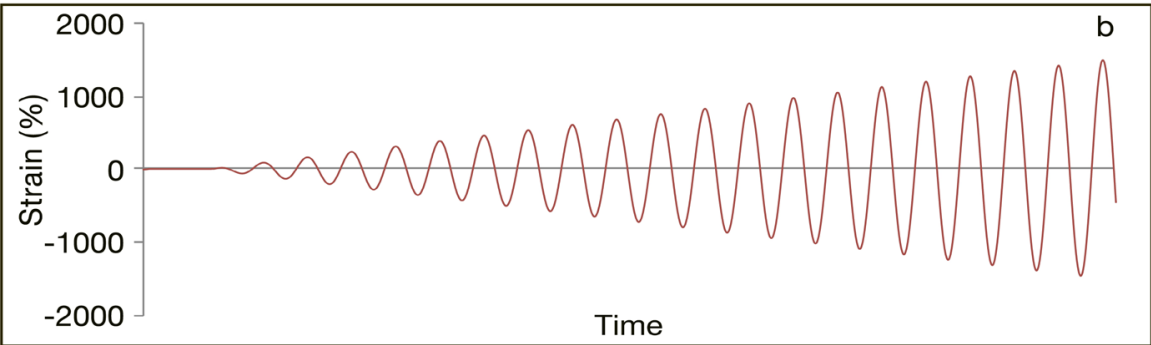

(b)

Figure 1. During a frequency sweep (a), the amount of deformation (strain) is constant while the frequency increases. During an amplitude/strain sweep (b), the frequency is constant, while the amount of deformation/amplitude/strain increases. 


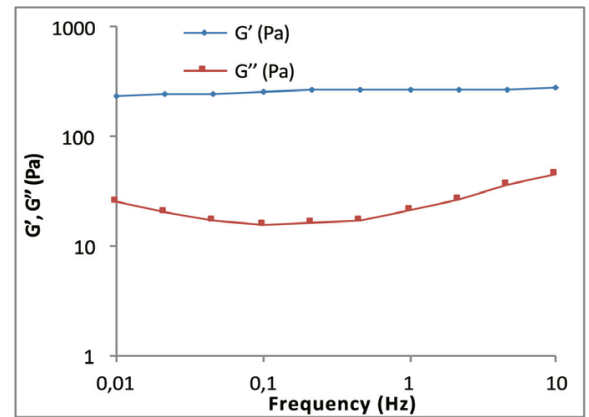

(a)

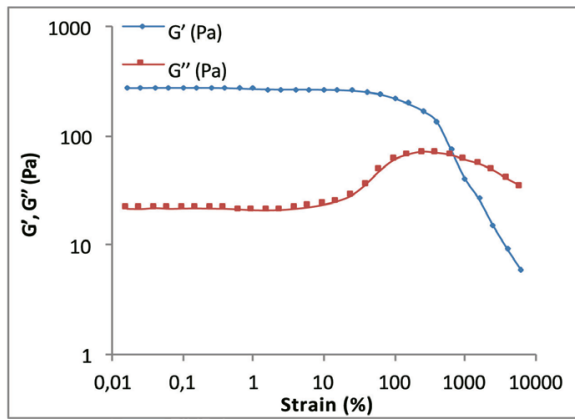

(b)

Figure 2. Frequency sweep (a) and amplitude sweep (b) of an HA filler.

However, there may be more information to be evaluated from the amplitude sweep. When the end of the LVR is reached, it means that the deformation is so large that the material can no longer return to its original shape, and starts behaving more like a liquid than a solid. In rheology, this point is referred to as the yield point.

A typical example of a material having a noticeable yield point is tomato ketchup, which moves frustratingly little until the bottle is shaken enough, resulting in the delivered portion of ketchup being larger than intended.

A material that can stand a large deformation before yielding can be described as being more stretchable, or flexible. In this way, the strain at the yield point can be considered an index of flexibility for the material.

The aim of this paper is to investigate how evaluation of the yield point in the amplitude sweep can be used as a measure of flexibility by studying a number of HA fillers produced using the optimal balance technology $\left(\mathrm{OBT}^{\mathrm{TM}}\right)$, in the US denoted the XpresHAn Technology ${ }^{\mathrm{TM}}$.

\section{Materials and Methods}

\subsection{Materials}

The HA-products tested in this study are shown in Table 1. The HA fillers studied are based on two different technologies, the OBT and the NASHA technology. The NASHA technology produces firmer gels based on molecular entanglements and small amounts of chemical crosslinking, with controlled particle sizes at different levels [12]. The OBT technology is based on four different levels of crosslinking, producing gels from very soft to intermediately firm, providing different levels of tissue support [13]. Since the OBT gels are also designed to vary in level of flexibility, these gels were selected to be investigated regarding their yield point, or index of flexibility. As a comparison RES and RLYF based on NASHA technology was included, being the firmest (highest G) in the product range.

\subsection{Test Methods}

The rheology measurement was performed in a sequence including a relaxation time of $30 \mathrm{~min}$, a frequency sweep from 10 to $0.01 \mathrm{~Hz}$ at $0.1 \%$ strain, followed by 
Table 1. Products tested in this study.

\begin{tabular}{cccc}
\hline $\begin{array}{c}\text { Product } \\
\text { (previous name) }\end{array}$ & Lot\# & Abbreviation & Technology \\
\hline $\begin{array}{c}\text { Restylane Defyne } \\
\text { (Emervel Deep Lidocaine) }\end{array}$ & 13,665 & RDEF & \\
$\begin{array}{c}\text { Restylane Volyme } \\
\text { (Emervel Volume Lidocaine) }\end{array}$ & 13,720 & RVOL & OBT \\
$\begin{array}{c}\text { Restylane Kysse } \\
(\text { Emervel Lips Lidocaine) }\end{array}$ & 13,827 & RKYS & \\
$\begin{array}{c}\text { Restylane Refyne } \\
\text { (Emervel Classic Lidocaine) }\end{array}$ & 13,869 & RREF & \\
$\begin{array}{c}\text { Restylane Fynesse } \\
\text { (Emervel Touch) }\end{array}$ & 12,886 & RFYN & NASHA \\
$\begin{array}{c}\text { Restylane Lyft Lidocaine } \\
\text { (Restylane Perlane Lidocaine) }\end{array}$ & 10,373 & RLYF & \\
Restylane Lidocaine & 10,634 & RES & \\
\hline
\end{tabular}

an amplitude sweep from $0.1 \%$ to $10000 \%$ (0.001 to 100) strain at $1 \mathrm{~Hz}$. The gap was $1 \mathrm{~mm}$ using a PP25 measuring system at $25^{\circ} \mathrm{C}$. The frequency sweep was evaluated for G' at $0.1 \mathrm{~Hz}$.

The amplitude sweep was first evaluated at $0.1 \%$ strain in order to verify that the applied frequency sweep strain was within the linear viscoelastic range. It was then evaluated at the cross-over point of the amplitude sweep, i.e. the point where G' and G "have the same value. At this point, the stress, strain and G (as G' and G' are identical) can be evaluated, their values denoted xStrain, xStress and $\mathrm{xG}$. For this study, xStrain was used for evaluation.

\section{Results}

In Figure 3 the strain value at the G'/G' cross-over point (xStrain) in the amplitude sweep is shown versus the firmness ( $G^{\prime}$ from the frequency sweep) for a number of OBT and NASHA products. The products cover a large range in G' and xStrain, where products with lower G' values tend to display higher xStrain values, and vice versa. The $\mathrm{xStrain}$ for OBT products range from below 1000\% for RDEF to above $2000 \%$ for RFYN. The NASHA reference products show low xStrain values, but also G' values about twice as high as the firmest OBT.

\section{Discussion}

\subsection{Method Setup}

Though there is a consensus on what the yield point is, the definition on where in the amplitude sweep this can be found may be a matter of discussion, see Figure 4. Generally, as soon as there is a change in the signal, e.g. a $1 \%$ decrease in the level of G', this would indicate the endpoint of the LVR (point a in Figure 4). Since there is always some noise in the signal, the change has to be of a certain magnitude in order to be correctly determined. When analyzing very soft 


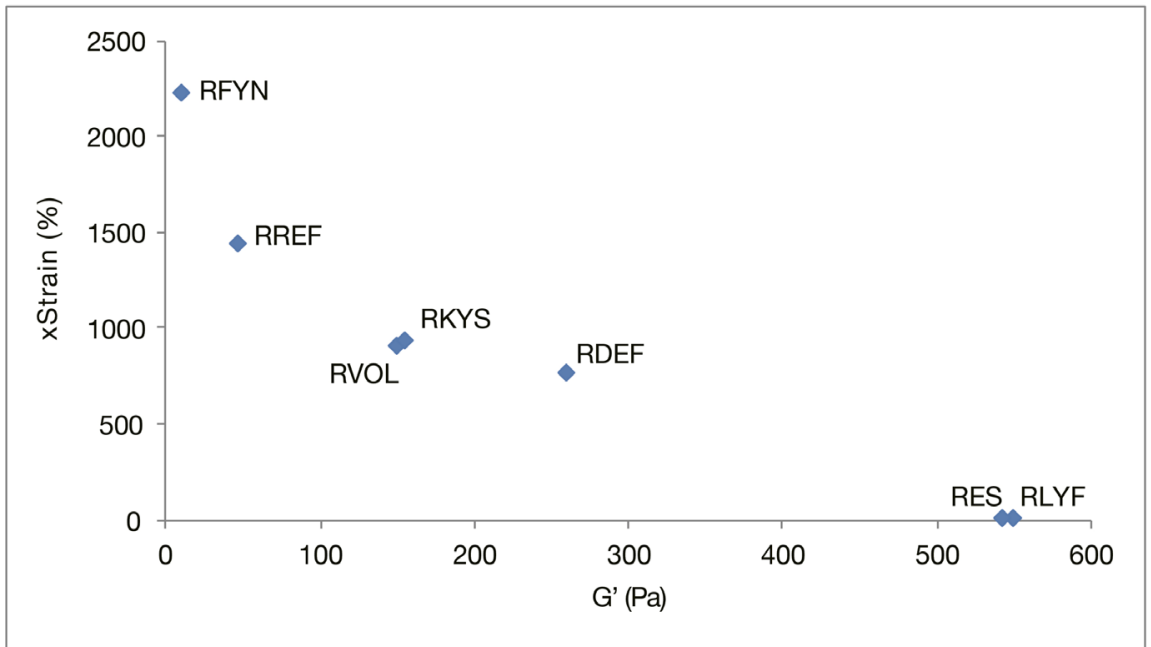

Figure 3. Flexibility (xStrain) versus firmness (G') for a number of OBT and NASHA products.

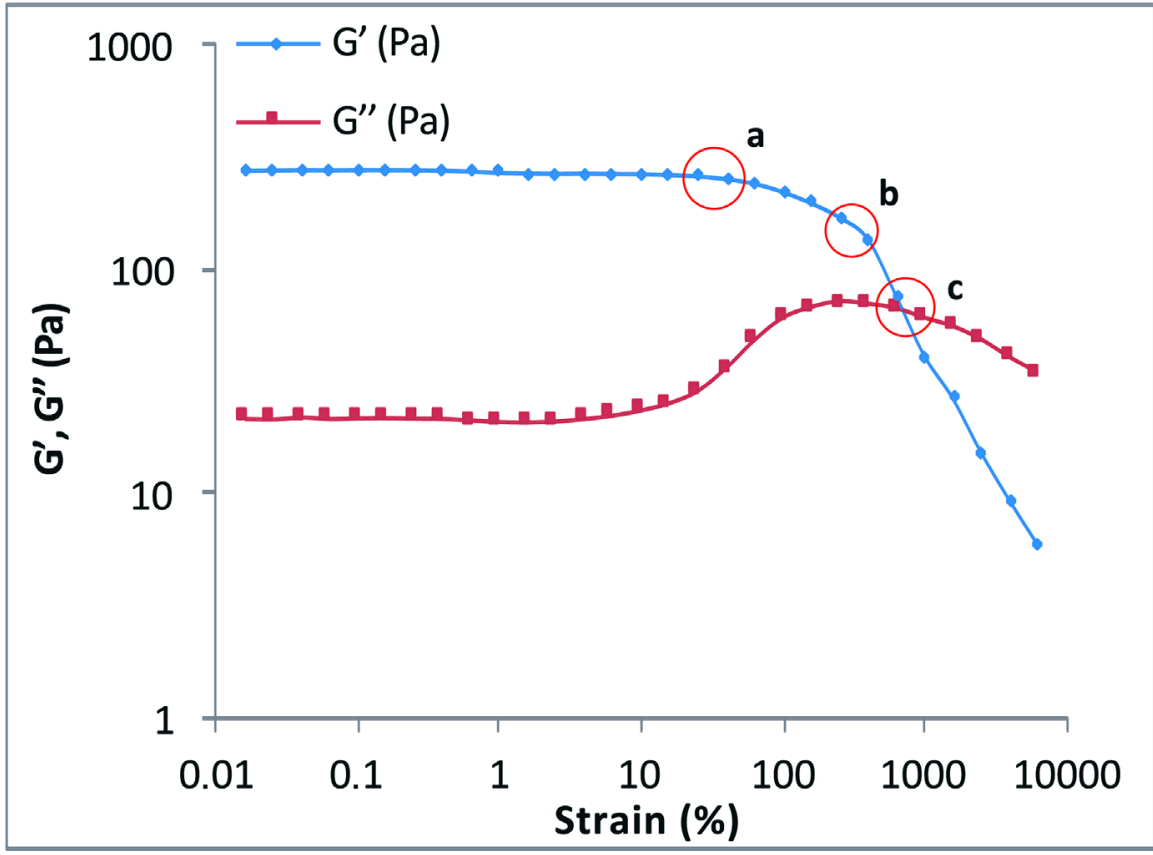

Figure 4. Amplitude sweep with possible yield points ( $a$ and $b$ ) and the cross-over point (c).

samples, giving a weaker signal, a larger deviation, e.g. a 10\% decrease in the level of G', has to be allowed in order not to incorrectly detect noise as the end of the LVR (point $b$ in Figure 4). An endpoint that is much easier to pinpoint exactly is the cross-over point, where G' and G' intersect (point c in Figure 4). Though this point may be considered to overestimate the yield point, its exactness and simplicity is a huge advantage.

Another topic is how to choose the frequency at which the amplitude sweep is performed. As given in the methods section, the frequency sweep was evaluated at $0.1 \mathrm{~Hz}$, a frequency relevant for small long term deformations in the clinical 
situation [14]. Using such a low frequency also for the amplitude sweep would result in excessively time-consuming measurements, due to the large number of measuring points required to precisely determine the cross-over point. The frequency sweep data have much less curvature, and therefore fewer data points are required in that case.

It can also be argued that since the frequency sweep is aimed at mimicking static conditions - the changes that occur in the tissue over days and months-a low frequency is appropriate.

The amplitude sweep is meant to reflect dynamic conditions-the movements in the tissue caused by facial expressions like smiling - a higher frequency may be more suitable. For these reasons, the amplitude sweep frequency was set to 1 $\mathrm{Hz}$.

The chosen measurement setup was considered to best combine a robust estimation of the flexibility with a reasonable measurement time.

\subsection{Flexibility and Level of Crosslinking}

The OBT family of products has previously been found to cover a large span of G' values as measured from a frequency sweep at small deformations [9]. In the current investigation, the OBT products demonstrated a range of flexibility measured as xStrain on the order of three times, with flexibility decreasing with increasing level of crosslinking.

The products in the OBT family are crosslinked using the same technology, but with different amounts of crosslinker added. Therefore it can be assumed that the main difference in the crosslinking structure is the distance between crosslinking points (Figure 5). From this follows that when the material is subjected to mechanical stress, the material with a larger distance between the crosslinking points will allow more deformation before the HA chains are fully stretched (Figure 6). This material will be perceived as more flexible, compared to a material with a smaller distance between crosslinking points.

Materials with a larger distance between the crosslinking points-everything else equal-will tend to be more flexible, and will also tend to be softer. The relation between softness and flexibility will, however, depend on the gel crosslinking technology. Two gels produced with different crosslinking technologies may demonstrate equal levels of softness, measured as G', but different levels of flexibility.

An exact and general correlation should not be expected, since the firmness derived from the small-deformation frequency sweep is a completely different property from the flexibility derived from the large-deformation amplitude sweep. Each property is measured separately at different conditions.

\subsection{Flexibility in the Clinical Situation}

The clinical relevance of gel firmness measured as G' has been discussed in a number of papers [1]-[13]. The flexibility measured as xStrain may prove to be 


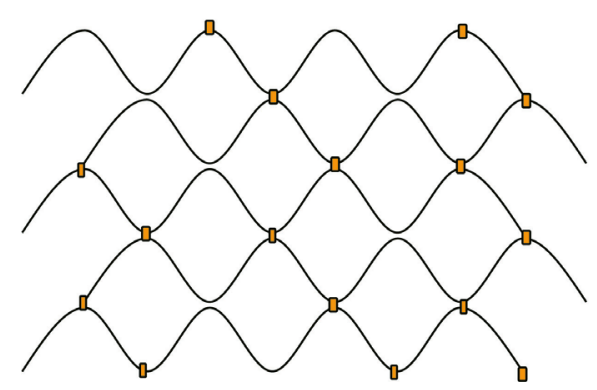

(a)

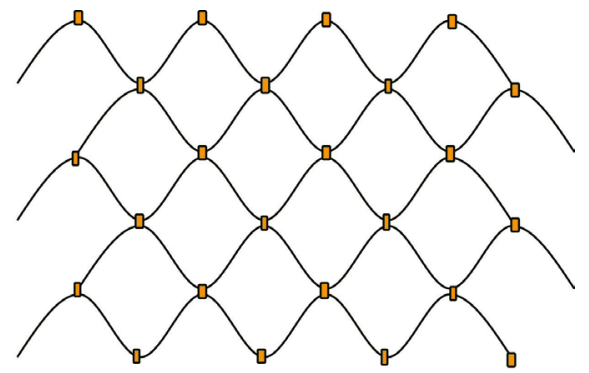

(b)

Figure 5. OBT products, with fewer (a) and more (b) crosslinking points, while in a relaxed state.

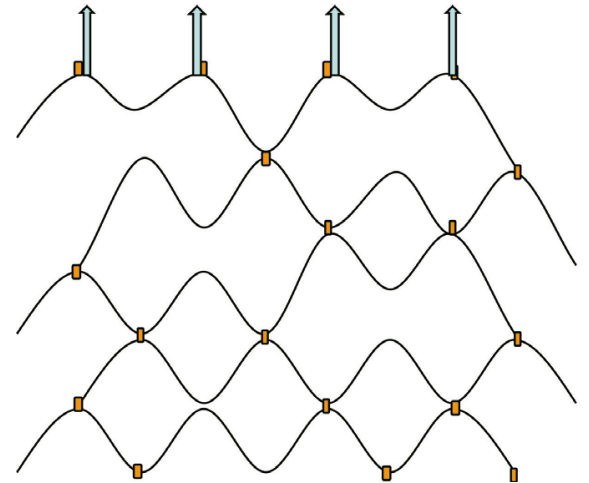

(a)

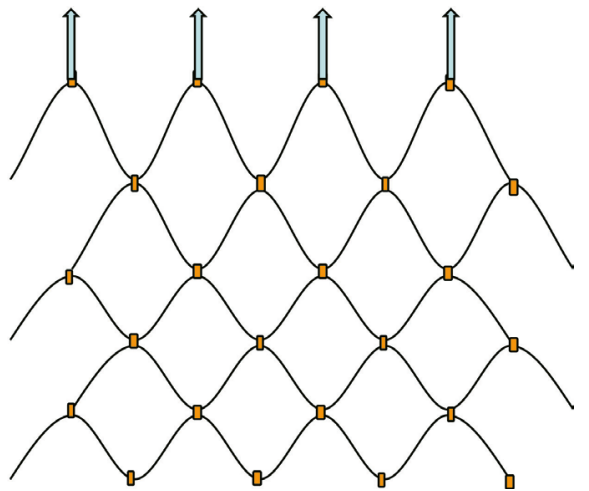

(b)

Figure 6. OBT products with fewer (a) and more (b) crosslinking points while in a stretched state.

an equally useful tool in the ongoing work of trying to predict the clinical behavior of HA filler products from their physical properties.

It would seem reasonable to argue that flexibility would be beneficial in dynamic areas of the face, allowing facial animation, while the firmness may be more important in areas with less movement. Of course, other factors such as tissue coverage of the individual patient must also be considered when balancing the need for flexibility versus firmness of an HA filler.

\section{Conclusion}

The flexibility of HA fillers can be accurately measured as xStrain derived from the G'/G' crossover point in the amplitude sweep. Measurement of the flexibility provides a more complete picture of the rheological properties of HA fillers as a complement to firmness measured as G'. The test results show that the OBT family of products covers a large range of flexibility. It can be argued that higher flexibility is beneficial in dynamic areas, facilitating natural facial expressions, while the firmness may be more important in areas with less movement.

\section{Disclosure}

Jan Åke Öhrlund is an employee of Galderma. 


\section{References}

[1] Pierre, S., Liew, S. and Bernardin, A. (2015) Basics of Dermal Filler Rheology. Dermatologic Surgery, 41, S120-126. https://doi.org/10.1097/DSS.0000000000000334

[2] Falcone, S.J., Doerfler, A.M. and Berg, R.A. (2007) Novel Synthetic Dermal Fillers Based on Sodium Carboxymethylcellulose: Comparison with Crosslinked Hyaluronic Acid-Based Dermal Fillers. Dermatologic Surgery, 33, S136-143.

https://doi.org/10.1097/00042728-200712001-00005

[3] Falcone, S.J. and Berg, R.A. (2008) Crosslinked Hyaluronic Acid Dermal Fillers: A Comparison of Rheological Properties. Journal of Biomedical Materials Research $A$, 87, 264-271. https://doi.org/10.1002/jbm.a.31675

[4] Kablik, J., Monheit, G.D., Yu, L., Chang, G. and Gershkovich, J. (2009) Comparative Physical Properties of Hyaluronic Acid Dermal Fillers. Dermatologic Surgery, 35, 302-312. https://doi.org/10.1111/j.1524-4725.2008.01046.x

[5] Sundaram, H., Voigts, B., Beer, K. and Meland, M. (2010) Comparison of the Rheological Properties of Viscosity and Elasticity in Two Categories of Soft Tissue Fillers: Calcium Hydroxylapatite and Hyaluronic Acid. Dermatologic Surgery, 36, 1859-1865. https://doi.org/10.1111/j.1524-4725.2010.01743.x

[6] La Gatta, A., Schiraldi, C., Papa, A. and De Rosa, M. (2011) Comparative Analysis of Commercial Dermal Fillers Based on Crosslinked Hyaluronan: Physical Characterization and in Vitro Enzymatic Degradation. Polymer Degradation and Stability, 96, 630-636. https://doi.org/10.1016/j.polymdegradstab.2010.12.025

[7] Stocks, D., Sundaram, H., Michaels, J., Durrani, M.J., Wortzman, M.S. and Nelson, D.B. (2011) Rheological Evaluation of the Physical Properties of Hyaluronic Acid Dermal Fillers. Journal of Drugs in Dermatology, 10, 974-980.

[8] Sundaram, H. and Cassuto, D. (2013) Biophysical Characteristics of Hyaluronic Acid Soft-Tissue Fillers and Their Relevance to Aesthetic Applications. Plastic and Reconstructive Surgery, 132, 5S-21S.

[9] Edsman, K.L., Wiebensjö, Å.M., Risberg, A.M. and Öhrlund, J.Å. (2015) Is There a Method That Can Measure Cohesivity? Cohesion by Sensory Evaluation Compared with Other Test Methods. Dermatologic Surgery, 41, S365-372. https://doi.org/10.1097/DSS.0000000000000550

[10] Hee, C.K., Shumate, G.T., Narurkar, V., Bernardin, A. and Messina, D.J. (2015) Rheological Properties and in Vivo Performance Characteristics of Soft Tissue Fillers. Dermatologic Surgery, 41, S373-381. https://doi.org/10.1097/DSS.0000000000000536

[11] Ascher, B., Rzany, B. and Sundaram, H. (Chairs) (2013) Fillers: From Characteristics to Recommendations. Consensus Panel Discussion. Consensus Panel Discus sion at: International Master Course on Aging Skin 2013 Annual Meeting, Paris, 31 January-3 February 2013.

[12] Edsman, K., Nord, L.I., Ohrlund, A., Lärkner, H. and Kenne, A.H. (2012) Gel Properties of Hyaluronic Acid Dermal Fillers. Dermatologic Surgery, 38, 1170-1179. https://doi.org/10.1111/j.1524-4725.2012.02472.x

[13] Segura, S., et al. (2012) Emervel: Full-Face Rejuvenation with a Range of Customized Hyaluronic Acid Fillers. Journal of Drugs in Dermatology, 11, 1.

[14] Lorenc, Z.P., Öhrlund, Å. and Edsman, K. (2017) Factors Affecting the Rheological Measurement of Hyaluronic Acid Gel Fillers. Journal of Drugs in Dermatology, 16, 611-617. 